\title{
Intraoperative stereotactic navigation for reconstruction in zygomatic-orbital trauma
}

\author{
Nyachhyon $\mathrm{P}^{1}$, Kim $\mathrm{PC}^{2}$ \\ 'Department of Plastic and reconstructive Surgery, Kathmandu Model Hospital, Nepal \\ ${ }^{2}$ Department of Plastic and Reconstructive Surgery, Daegu Catholic Medical Center, South Korea
}

\section{ABSTRACT}

Reconstruction of the maxillofacial skeleton after traumatic injury is not always straightforward because of its three dimensional complex anatomy and aesthetic significance. In case of zygomaticorbital injury induced by trauma resulting in enophthalmos, the patient is affected on both function and cosmesis. To prevent diplopia or conspicuous asymmetry, exact correction of the orbital symmetry is required. The aim of this case report is to demonstrate the use of an intraoperative image guided navigation system for identification of adequate reconstruction of the orbital floor and orbital symmetry in zygomatic-orbital fracture. Navigation-guided open reduction of a zygomatic-orbital complex fractures with orbital floor reconstruction can be regarded as a valuable treatment option for this potentially complicated procedure. This case demonstrated the use of the non-ionizing, noncontact, navigation system in intraoperative procedure and clinical monitoring to identify the correct position and symmetry in complex orbital floor reconstruction.

Keywords: intraoperative navigations, orbital reconstruction, zygomatico-orbital fracture,

\section{INTRODUCTION}

Management of zygomatic-orbital fractures requires a thorough ophthalmic evaluation and precise imaging. When orbital deformity is present in the form of enophthalmos, exophthalomos or dystopia, it becomes a further challenge to achieve three dimensional orbital symmetry. Many reports in the literature cite difficulty in obtaining adequate reduction, even with direct observation. We usually rely on the immediate adjacent nondisplaced bone for restoration of contour but in complex fractures, the adjacent bone or the bone in the contralateral side may be lost or comminuted, leaving little guidance to the 3-dimensional skeletal architecture and resulting in facial asymmetry and disfigurement postoperatively. Diagnostic advances such as new surgical and imaging techniques have dramatically improved both the functional and aesthetic outcome of reconstructions. Intraoperative $\mathrm{CT}$ has been used to determine the accuracy of orbital reconstruction.
However it is associated with high staffing, equipment cost and extra radiation exposure. With the advent of navigational surgery, a technical revolution has been promoted to benefit treatment to reduce overall operation time, making surgery more reliable and allowing safer manipulations in close proximity to delicate structure ${ }^{1}$. In 1908 Sir Victor Horsley and Robert Clarke $^{2}$ of the University College, London first described a machine for accurate localization within the cranial cavity in animals and later Spiege ${ }^{3}$ used framed stereotaxy in humans in 1947 . The navigation system has been used successfully in neurosurgery, orthopaedics and otolarygology for intraoperative corroboration using preoperative radiologic images. Surgical navigation has been described in a wide variety of maxillofacial procedures such as operations on the skull base, paranasal sinus and orbit to remove foreign bodies and for orthognathic and implant surgeries ${ }^{4}$.

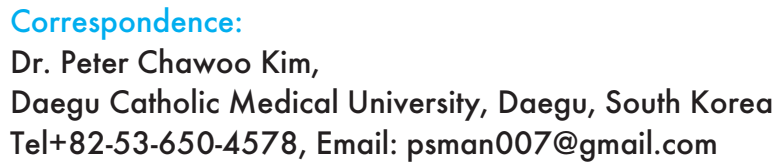


The image guided navigation system is a relatively useful tool that can assist surgeons to guide adequate reduction of the fractured segments and reconstruction of the maxillofacial skeleton. We report the method of intraoperative use of a navigation system for localization of displaced facial skeletal segments during reduction and internal fixation of zygomatic-orbital fractures and restoration of orbital symmetry.

\section{CASE REPORT}

A 25-year-old female presented with a sunken eye on the left side in the horizontal and vertical plane two years and four months after having undergone surgery for a zygomatico-maxillary complex fracture, involving the orbital floor. The patient was affected by double vision and a noticeable defective globe position as dystopia, with deepening of the supratarsal fold. The zygomatic-orbital anatomy was assessed in multiplanar (axial, coronal, sagittal) and 3-dimensional computed tomography (CT) hard-tissue view. In planning the correction of the globe position, the patient's preoperative CT images were processed by using Invivo 5 software to obtain mirror images of the normal side. An impression of the maxilla was taken and was used to fabricate a plastic model. A vacuum-formed plastic splint was constructed with omnivac over the plaster model. The base components for attaching the CT markers were polymerized and buried into the base of the splint as reference for the navigation system. The CT scan was achieved with the splint-attaching screw marker and was used to fabricate a 3D rapid prototype plastic model. Preoperative surgical planning based on the 2D life-size photos and 3D images was accomplished.

The comparison of orbital symmetry revealed enophthalmos of $6.3 \mathrm{~mm}$ on relative Hertel's exophthalmometry and cross sectional CT image. The decision was made to reconstruct the orbital floor with a Synpor (Polyethylene Titanium mesh) and correction of the medial wall defect with a particulate bone graft. The navigation system VectorVision2 (BrainLAB, Heimstetten, Germany) was used. The registration of the patient' position was done in relation to the image data set. A Mayofield head set with three steel prongs was mounted on the patient's head. A light emitting diodes (LED) array was securely attached to the patient. At the start of the operation, the reference points on the plastic dental splint were sequentially touched with an instrument fitted with LED and they were simultaneously digitized in the image data. When the setup was complete, a tracking instrument could be moved during the operation while watching the corresponding movement of the instrument in the monitor. The accuracy of this referencing method, consisting of a cortical fixed reference system and surface scanning, was checked by anatomic landmarks.

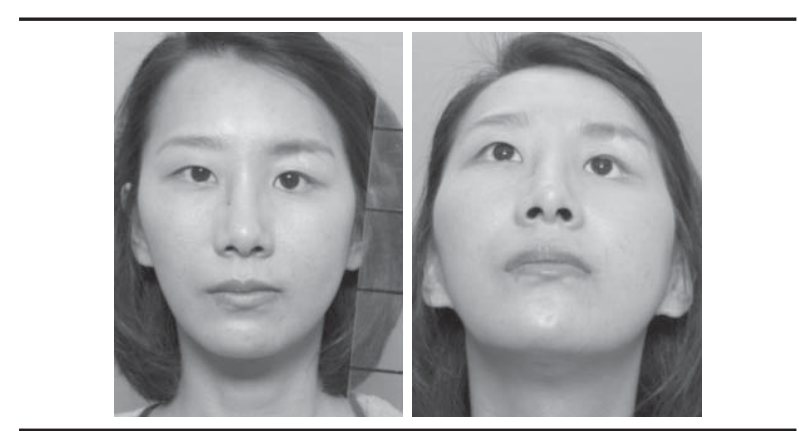

Figure 1. Preoperative frontal (left) and low basal view (right) Frontal and low basal views show marked dystopia and enophthalmos in the left side

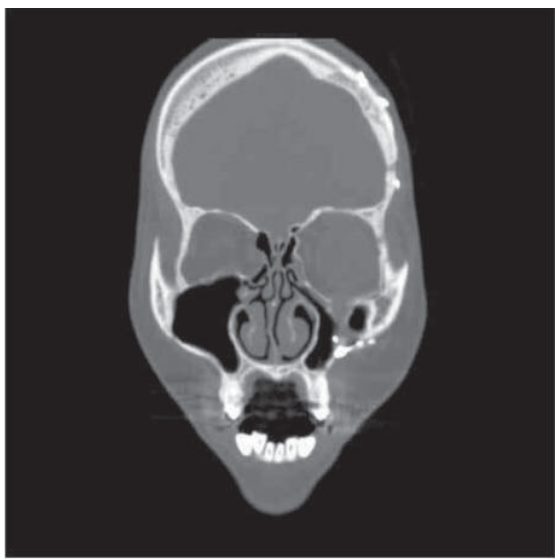

Figure 2. Coronal section of preoperative CT figures The floor of the left orbit is more lowered than that of the right side. The soft tissue has drooped through the floor.

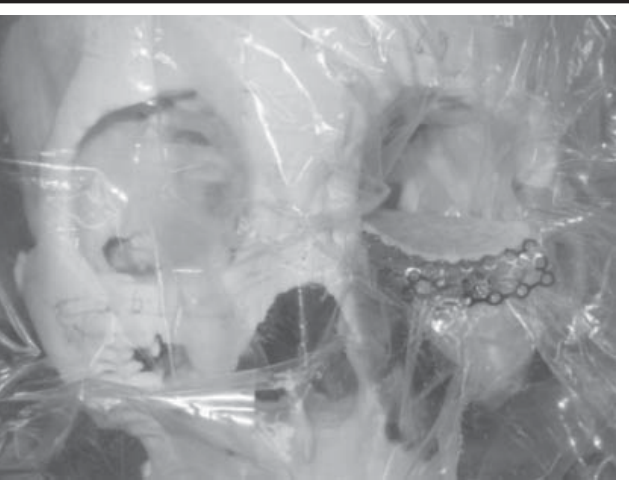

Figure 3. Preadaptation of titanium mesh embedded in Synpor ${ }^{\circledR}$ The D Titanium mesh embedded is preformed with the rapid protoyped - 3D dimensional model.

The patient underwent open reduction and orbital floor reconstruction under the guidance of the navigation system. The infra orbital rim and the floor of the orbit were exposed through the subciliary approach. The defect in the floor of the orbit was exposed and the extent of the defect correlated with the navigation. The 
internal orbit was reconstructed with the preformed Titanium embedded in UMHWPE (ultra high molecular weight polyethylene) mesh (SynPOR Synthes, Switzerland). The external orbital frame was reduced and stabilized using $1.5-\mathrm{mm}$ titanium plates and screws. Intraoperative navigation was used to assess the accuracy of the restored internal and external orbital anatomy by assessing various points on the digitalized images.

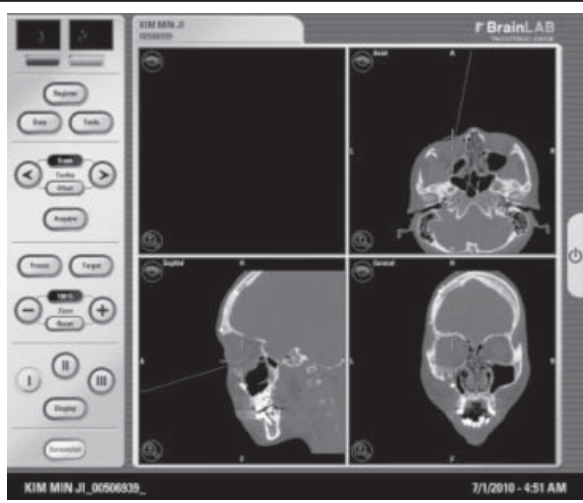

Figure 4. Secondary reconstruction for enophthalmosintraoperative navigation. The criss-cross (green color) in the monitor which indicated the tip of probe shows the level of titanium mesh embedded in Synpor ${ }^{\circledast}$

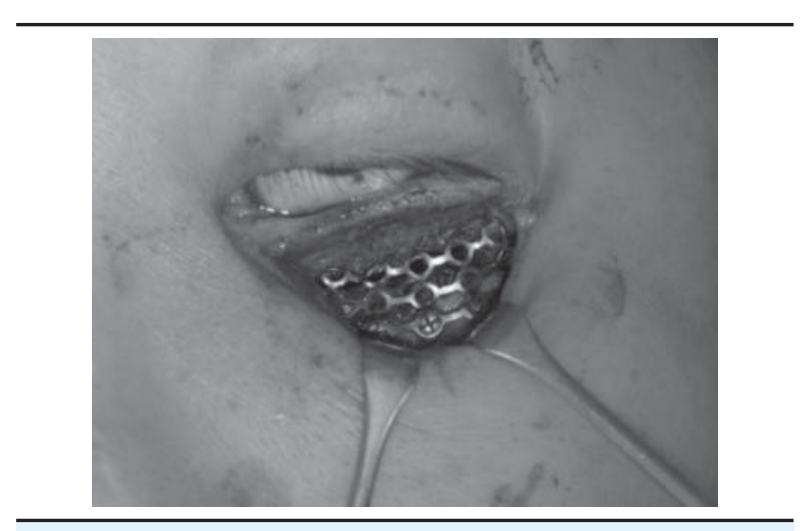

Figure 5. Intraoperative view Subciliary incision allowed titanium mesh reconstruction

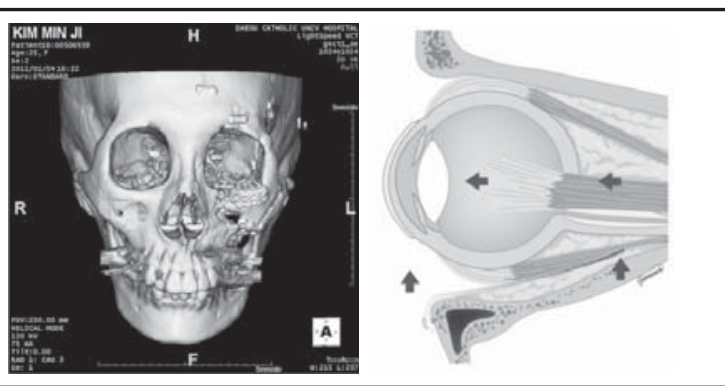

Figure 6. Frontal view of 3D computer tomogram six months after surgery Titanium mesh is well positioned and the zygoma osteotomy site is well-united

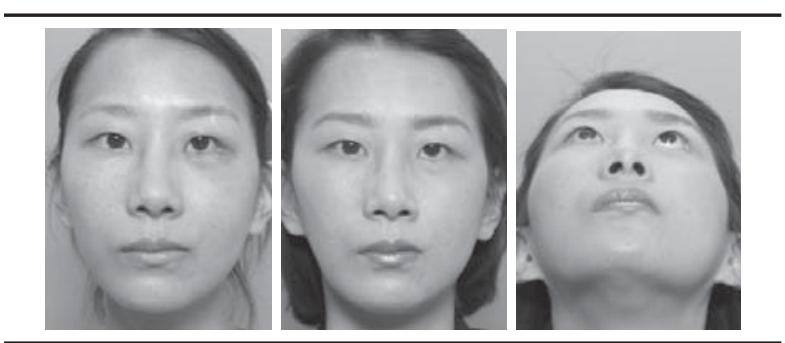

Figure 7. Postoperative view at two weeks and six month after srugery

The left photo shows the frontal view two weeks after surgery. The middle photo shows the frontal view six months postoperative. Dystopia is well corrected and mild scleral show is shown. The right photo shows the high basal view at six months postoperatively. Enophthalmos has been corrected

At the end of surgery, there was a difference of 1.2 $\mathrm{mm}$ between two eyeball globe protrusions. The reduction was checked by postoperative computed tomography scans. The preoperative and postoperative images were compared and subjectively analyzed. Two weeks after surgery, the symptoms associated with the orbital floor defects (double vision) were found to be eliminated and the postoperative facial appearance of the patient had clearly improved. Postoperative CT scans and photographs revealed excellent correction of enophthalmos, significant improvement in symmetry of the nasoethmoid complex and symmetrical reconstruction of the zygomaticomaxillary complex.

\section{DISCUSSION}

Navigation is a surgical adjunct and its purpose is to improve the safety and outcome of existing surgical procedures ${ }^{4}$. Computer-aided surgery is improving the surgical outcomes in maxillofacial reconstruction. Several recent articles report clinical outcomes using computer-aided surgery, investigate the precision of various intraoperative optical navigation systems, compare several registration strategies and examine the impact of natural facial asymmetry on planning and reconstruction ${ }^{5}$. The new techniques of navigation surgery allow us to interactively use the threedimensional image data of the patient during surgery. It is the primary goal of computer-assisted surgery to support the surgeon during diagnosis, operational planning and intraoperative navigation.

The computer aided navigation system has been widely used in neurosurgery, orthopaedics and ortolaryngological surgeries. With the introduction of the computer assisted navigation system, the interactive, intraoperative application of three dimensional image data can be realized so that precision achieved during planning phase can be transferred to the patient. We can 
thereby decrease operational risk and surgical duration, which in turn results in considerable stress reduction for the patient ${ }^{6}$. In maxillofacial surgery, one of the best examples of the benefit of navigation is in the field of secondary orbital reconstruction.

Orbitozygomatic fractures pertain to the most common injuries in craniofacial trauma patients. Accurate fracture reduction is of high importance for a successful outcome and the study shows the potential benefit of surgical navigation in orbitozygomatic fracture reduction.

A variety of registration methods including anatomical landmarks, miniscrews inserted into the bone preoperatively and surface based concept is proposed ${ }^{7}$. These reference points are used for coordinate transfer between the preoperative data set and the surgical site. Inventions in the field of non-invasive registration have led to widespread use of computer-assisted simulation and navigational surgery in this field. New methods of non-invasive referencing in the field of cranio-maxillofacial surgery have been described in the literature ${ }^{8}$.
A major drawback of the navigation system is a high consumption of human financial resources and technical expertise ${ }^{6}$. A larger clinical series with a long-term follow-up will be needed to determine reproducibility and cost-effectiveness. Navigation is likely to continue to have a role in orbital reconstruction. The complex and individual nature of the cases that benefit most make it hard to compare outcome with and without navigation and debate is likely to continue as to whether the cost of these systems is justified ${ }^{6}$

\section{ACKNOWLEDGEMENTS}

We gratefully acknowledge and thank Gemma Seung Jung Son (BA), the medical illustrator in our department. Miss Gemma produced the illustrations in this article, visualized works of art that only artists like she can create.

\section{REFERENCES}

1. Hassfeld S, Zoller J, Albert FK, et al. Preoperative planning and intraoperative navigation in skull base surgery. J Craniomaxillofac Surg. 1998; 26:220.

2. Horsley V, Clarke RH. The structure and function of cerebellum examined by a new method. Brain 1908; 51:45124.

3. Spiegel EA, Wycis HT, Marks M, Lee AJ. Stereotaxic apparatus for operations on the human brain. Science 1947; 106:349-50.

4. Collyer J. Stereotactic navigation in oral and maxillofacial surgery. Br J Oral Maxillofac Surg. 2010; 48: 79-83.
5. Hassfeld S, Muhling J. Computer assisted oral and maxillofacial sugery-a review and an assessment of technology. Int. Oral Maxillofac. Surg. 2001; 30:2-13.

6. Max H, Christian HR, Rainer S. Indications and limitations of intraoperative navigation in maxillofacial surgery. J Oral Maxillofac Surg. 2004; 62: 1059-1063.

7. Rudiger M, Stefan H, Tim L, Joachim M. Laser scan based navigation in cranio-maxillofacial surgery. Journal of craniomaxillofacial surgery. 2003; 31: 267-277.

8. Heiland M, Habermann CR, Schmelzle R. Indications and limitations of intraoperative navigation in maxillofacial surgery. J Oral Maxillofac Surg. 2004 Sep;62(9):1059-63. 